\title{
Suppression of deterministic and stochastic extreme desynchronization events using anticipated synchronization
}

\author{
Jordi Zamora-Munt, Claudio R. Mirasso, and Raul Toral \\ Instituto de Física Interdisciplinar y Sistemas Complejos, IFISC (CSIC-UIB), E-07122 Palma de Mallorca, Spain
}

(Received 15 October 2013; revised manuscript received 19 December 2013; published 31 January 2014)

\begin{abstract}
We numerically show that extreme events induced by parameter mismatches or noise in coupled oscillatory systems can be anticipated and suppressed before they actually occur. We show this in a main system unidirectionally coupled to an auxiliary system subject to a negative delayed feedback. Each system consists of two electronic oscillators coupled in a master-slave configuration. Extreme events are observed in this coupled system as large and sporadic desynchronization events. Under certain conditions, the auxiliary system can predict the dynamics of the main system. We use this to efficiently suppress the extreme events by applying a direct corrective reset to the main system.
\end{abstract}

DOI: 10.1103/PhysRevE.89.012921

PACS number(s): 05.45.Gg, 05.45.Xt, 05.40.Ca, 89.75.-k

\section{INTRODUCTION}

Extreme events can be defined as those whose amplitude or duration, being much larger than the average, is representative of the tail of a probability distribution [1]. This kind of event has been reported in different disciplines ranging from climatology to optics, population dynamics, and the economy [2-6], and its study has attracted the interest of researchers in recent years due to the possible destructive effects of these events. Many efforts have been devoted to understanding their origin, predicting when and where they will appear, and, if possible, suppressing them [7-9].

Some of these extreme events, being irregular and sporadic, are considered unpredictable: they appear spontaneously without a precursor or any clue as to how they will evolve [10]. However, recent studies have shown that some extreme events can appear in the context of deterministic dynamics [11,12], suggesting that they can be predicted. Meanwhile, it is not clear if truly random extreme events (induced by some source of noise) can also be predicted and suppressed, and this still constitutes a challenge in current research.

Extreme stochastic or deterministic events can be found in coupled systems as sporadic desynchronization intervals induced by noise or parameter mismatches. These desynchronization events are also known as "bubbling" and have been observed and intensively studied, for instance, in semiconductor lasers [13,14] and electronic circuits [15]. Recently, Cavalcante and co-workers [16] studied bubbling events of two unidirectionally coupled electronic circuits. For small parameter mismatches or under the influence of noise, the sizes of the small desynchronization events are distributed according to a power law. Nevertheless, the largest desynchronization events are more frequent than one could expect by extrapolating the power-law distribution of the small ones. This property characterizes a particular type of extreme events known as dragon kings (DKs) [8].

A plausible method to predict DKs is by anticipating the events. This could be done through the anticipated synchronization that can be achieved by unidirectionally coupling an auxiliary system to a main system [17]. Under certain conditions the dynamics of the auxiliary system is identical to that of the main one but advanced by a certain time. It has been demonstrated that this scheme efficiently predicts chaotic and excitable dynamics [18-20]. Furthermore, it has been used to suppress noise-induced spikes [21,22] by applying an appropriate external input to the target system.

The aim of this paper is to elucidate whether it is possible to anticipate extreme desynchronization events and suppress them by using anticipated synchronization. The proposed method allows us to know in advance the dynamics of a target system and apply corrective resets to keep these events under a safety amplitude.

This paper is organized as follows: In Sec. II we describe the system used to obtain the extreme events, the coupling scheme, and the parameters that will lead to stable anticipated synchronization. In Sec. III we study the cases of deterministic events (Sec. III A) induced by parameter mismatch and stochastic events induced by an external noise source (Sec. III B). Finally, in Sec. IV we summarize our main results.

\section{PREDICTION OF EXTREME EVENTS}

The general coupling scheme we use to obtain anticipated synchronization consists of two unidirectionally delaycoupled dynamical systems as introduced in [17],

$$
\begin{gathered}
\dot{\mathbf{x}}=f(\mathbf{x}(t)), \\
\dot{\mathbf{y}}=f(\mathbf{y}(t))+\mathbf{C}[\mathbf{x}(t)-\mathbf{y}(t-\tau)],
\end{gathered}
$$

where $\mathbf{x}$ is the main system and $\mathbf{y}$ is the auxiliary system. Additionally, the overdot represents a time derivative, $\mathbf{C}$ is the matrix of coupling strengths, and $\tau$ is the feedback delay time. In some parameter regions the solution $\mathbf{x}(t+\tau)=\mathbf{y}(t)$ is stable, such that the system $\mathbf{y}$ predicts the behavior of $\mathbf{x}$ a time $\tau$ in advance.

In our particular application the main system $\mathbf{x}=$ $\left(V_{M 1}, v_{M 1}, I_{M 1}, V_{S 1}, v_{S 1}, I_{S 1}\right)$, consists of two unidirectionally coupled electronic oscillators in a master-slave configuration [16] as shown in Fig. 1. The dynamical evolution of the main master system $M_{1}$ is given by

$$
\dot{V}_{M_{1}}=\frac{V_{M_{1}}}{R_{1, M_{1}}}-g_{M_{1}}\left[V_{M_{1}}-v_{M_{1}}\right]
$$




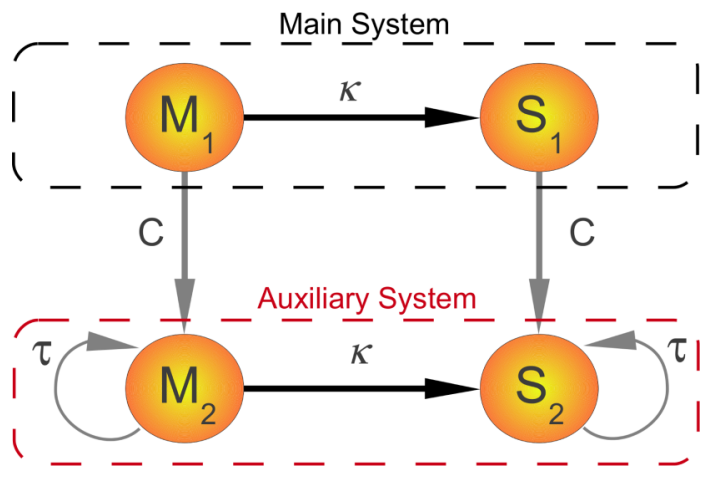

FIG. 1. (Color online) Coupling scheme used for anticipated synchronization. Each subsystem consist of two unidirectionally coupled oscillators in a master-slave configuration. The main system is unidirectionally coupled to the auxiliary system according to Eqs. (1) and (2).

$$
\begin{gathered}
\dot{v}_{M_{1}}=g_{M_{1}}\left[V_{M_{1}}-v_{M_{1}}\right]-I_{M_{1}}, \\
\dot{I}_{M_{1}}=v_{M_{1}}-R_{4, M_{1}} I_{M_{1}},
\end{gathered}
$$

and it is unidirectionally coupled to the main slave $S_{1}$ whose dynamics is described by

$$
\begin{gathered}
\dot{V}_{S_{1}}=\frac{V_{S_{1}}}{R_{1, S_{1}}}-g_{S_{1}}\left[V_{S_{1}}-v_{S_{1}}\right], \\
\dot{v}_{S_{1}}=g_{S_{1}}\left[V_{S_{1}}-v_{S_{1}}\right]-I_{S_{1}}+\kappa\left(v_{M_{1}}-v_{S_{1}}\right), \\
\dot{I}_{S_{1}}=v_{S_{1}}-R_{4, S_{1}} I_{S_{1}},
\end{gathered}
$$

where $g_{j}$ with $j=M_{1}$ or $S_{1}$ has the form

$$
g_{j}[V]=\frac{V}{R_{2, j}}+I_{r, j}\left(e^{\alpha_{f, j} V}-e^{-\alpha_{r, j} V}\right) .
$$

The coupling from $M_{1}$ is introduced in Eq. (7) with a coupling strength $\kappa$. We arbitrarily couple the master and the slave oscillators through the variable $v_{S_{1}}$ because synchronization is less stable than a coupling through $V_{S_{1}}$ [15].

Synchronization between $M_{1}$ and $S_{1}$ can be visualized via a distance $x_{\perp}$ in the phase space, defined as

$$
x_{\perp}=\left|V_{M_{1}}-V_{S_{1}}\right|+\left|v_{M_{1}}-v_{S_{1}}\right|+\left|I_{M_{1}}-I_{S_{1}}\right| .
$$

$x_{\perp}$ equals zero if $M_{1}$ and $S_{1}$ are identically synchronized and is larger than zero during a desynchronization event. In the absence of noise and when the parameter values of $M_{1}$ and $S_{1}$ are identical, the two oscillators identically synchronize, leading to $x_{\perp}=0$ at all times. For a small parameter mismatch between $M_{1}$ and $S_{1}$ sporadic desynchronization events of all sizes can occur. This is shown in Fig. 2 (solid black line) where the largest events (roughly $x_{\perp}>2$ ) are the so-called dragon kings previously introduced. From now on we consider deterministic bubbling induced by parameter mismatch, while the stochastic bubbling induced by noise will be considered in Sec. III B.

For prediction and control purposes we introduce an auxiliary system $\mathbf{y}=\left(V_{M 2}, v_{M 2}, I_{M 2}, V_{S 2}, v_{S 2}, I_{S 2}\right)$. The auxiliary system also consists of two unidirectionally coupled electronic
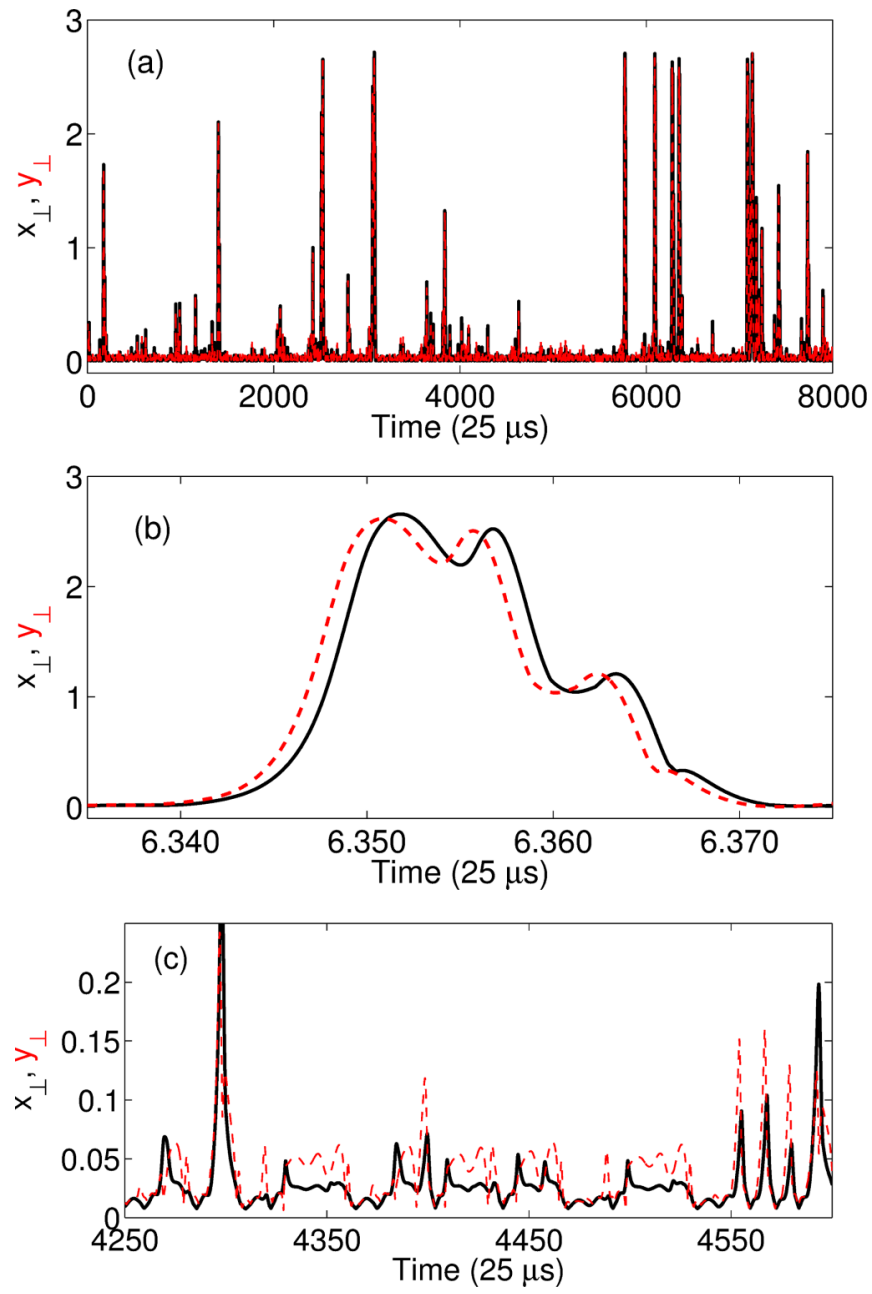

FIG. 2. (Color online) (a) Time trace of $x_{\perp}$ for the main system (black solid line) and $y_{\perp}$ for the desynchronization events predicted by the auxiliary system (dashed red line). (b) A magnification of an extreme event in the main system (solid black line) and the event predicted by the auxiliary system (dashed red line). (c) A magnification of the small desynchronization events in the main system (solid black line) and the events predicted by the auxiliary system (dashed red line). $\tau=1, C=0.75$, and the other parameters are listed in Table I. This and other figures have been obtained by a numerical integration of the governing equations of the system using a Runge-Kutta method of second order with a small enough time step $\left(d t=10^{-4}\right)$ to ensure the convergence of the method. Nevertheless, we have also verified that the results obtained are statistically independent for larger time steps.

oscillators in a master-slave configuration. Furthermore, it receives an input from the main system and is also subject to a negative delayed feedback as shown in Fig. 1. The dynamical evolution of the auxiliary master $M_{2}$ is given by

$$
\begin{gathered}
\dot{V}_{M_{2}}=\frac{V_{M_{2}}}{R_{1, M_{2}}}-g_{M_{2}}\left[V_{M_{2}}-v_{M_{2}}\right]+C\left[V_{M_{1}}-V_{M_{2}}(t-\tau)\right] \\
\dot{v}_{M_{2}}=g_{M_{2}}\left[V_{M_{2}}-v_{M_{2}}\right]-I_{M_{2}} \\
\dot{I}_{M_{2}}=v_{M_{2}}-R_{4, M_{2}} I_{M_{2}}
\end{gathered}
$$


TABLE I. Parameters of the master and slave oscillators in the main $\left(M_{1}\right.$ and $\left.S_{1}\right)$ and auxiliary $\left(M_{2}\right.$ and $\left.S_{2}\right)$ systems where the time is normalized to $T=25 \mu \mathrm{s}$. They correspond to a parameter mismatch of $\sim 1 \%-2 \%$ with respect to $M_{1}$.

\begin{tabular}{lcccc}
\hline \hline Parameter & $M_{1}$ & $S_{1}$ & $M_{2}$ & $S_{2}$ \\
\hline$I_{r}(\mu \mathrm{A})$ & 22.5 & 22.4 & 22.3 & 22.6 \\
$\alpha_{f}$ & 11.6 & 11.5 & 11.7 & 11.8 \\
$\alpha_{r}$ & 11.57 & 11.71 & 11.8 & 11.43 \\
$R_{1}$ & 1.298 & 1.308 & 1.32 & 1.28 \\
$R_{2}$ & 3.44 & 3.47 & 3.41 & 3.5 \\
$R_{4}$ & 0.193 & 0.195 & 0.191 & 0.2 \\
$\kappa$ & & 4.6 & & 4.6 \\
\hline \hline
\end{tabular}

Similarly, the equations for the auxiliary slave system $S_{2}$ have the form

$$
\begin{gathered}
\dot{V}_{S_{2}}=\frac{V_{S_{2}}}{R_{1, S_{2}}}-g_{S_{2}}\left[V_{S_{2}}-v_{S_{2}}\right]+C\left[V_{S_{1}}-V_{S_{2}}(t-\tau)\right], \\
\dot{v}_{S_{2}}=g_{S_{2}}\left[V_{S_{2}}-v_{S_{2}}\right]-I_{S_{2}}+\kappa\left(v_{M_{2}}-v_{S_{2}}\right), \\
\dot{I}_{S_{2}}=v_{S_{2}}-R_{4, S_{2}} I_{S_{2}},
\end{gathered}
$$

where the nonlinear functions $g_{M_{2}}$ and $g_{S_{2}}$ are given by Eq. (9), $C$ is the coupling strength, and $\tau$ is the feedback delay time. The main system is coupled to the auxiliary system through the variable $V$ because the synchronization is more stable than a coupling through $v$ [15]. Following the previous notation, a distance $y_{\perp}$ between $M_{2}$ and $S_{2}$ in the phase space is defined by

$$
y_{\perp}=\left|V_{M_{2}}-V_{S_{2}}\right|+\left|v_{M_{2}}-v_{S_{2}}\right|+\left|I_{M_{2}}-I_{S_{2}}\right| .
$$

The accuracy of the anticipation of extreme events also depends on the parameter mismatch. Table I uses parameters that differ by $\sim 1 \%-2 \%$, and for these values the auxiliary system can predict accurately the extreme events as shown in Fig. 2 (dashed red line). The accuracy $\rho$ of the prediction is shown in Fig. 3 in the coupling strength and delay time parameter space $(C, \tau), \rho$ is calculated as the ratio of the number of peaks in the distance $y_{\perp}$ for the auxiliary divided

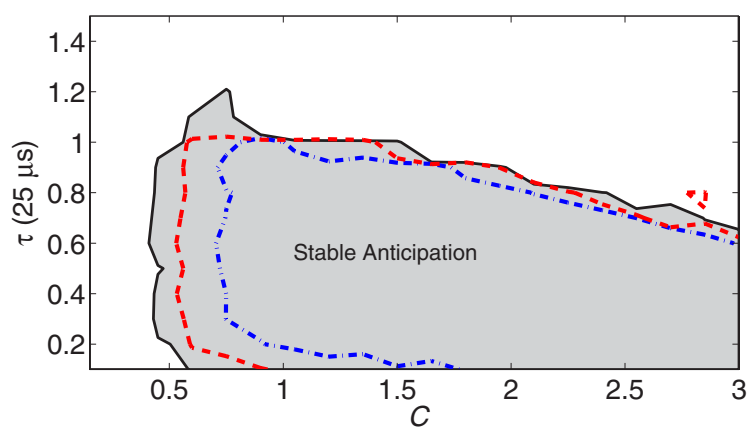

FIG. 3. (Color online) Boundary of stable anticipated synchronization with an accuracy, $\rho$, larger than 0.9 in the plane $(C$, $\tau$ ). The parameter mismatch between the main and the auxiliary systems is $\sim 2 \%$ (solid black line), $\sim 5 \%$ (dashed red line), and $\sim 10 \%$ (dash-dotted blue line). The boundaries were averaged using 4 different sets of parameters. by the number of peaks in the distance $x_{\perp}$ of the main system. Since we are interested in predicting the largest events, we consider in the calculation of $\rho$ only peaks larger than 0.3 times the absolute maximum in a realization and discard the peaks smaller than this value. Actually, small bubbling events can be less accurately predicted than the large ones [Fig. 2(c)], since they are of the same order of magnitude as the desynchronization between the main and the auxiliary systems. As in other examples [17,21,22], stable anticipated synchronization occurs in a wide range of parameters. In Fig. 3, the largest prediction time $\tau \sim 1$ occurs when the coupling strength is $C=0.75$. In what follows and to study the suppression of extreme events in the subsequent sections we fix $\tau=1$ and $C=0.75$ [23]. We verified that our predictive scheme also leads to stable anticipated synchronization when the auxiliary system has a wider parameter mismatch, e.g., for sets of parameters with a 5\% (Fig. 3, dashed red line) and a 10\% (Fig. 3, dash-dotted blue line) mismatch with respect to the main system.

Note that the coupling between main and auxiliary systems acts unidirectionally, and the main system is not affected by its existence, so that its dynamics is not altered at all by the presence or not of the auxiliary system.

\section{SUPPRESSION OF EXTREME EVENTS}

As previously discussed, the desynchronization or bubbling events in the main system can be deterministic, due to small mismatches in the parameters, or stochastic, due to the presence of noise even when the master and the slave systems are identical $[13,14]$. In the following section we study the suppression of large deterministic desynchronization events induced by a parameter mismatch, while in Sec. III B we consider identical units under the influence of noise.

\section{A. Deterministic extreme events induced by parameter mismatch}

In order to avoid bubbling events and to stabilize the synchronized state of the main system we consider a simple method of control based on corrective signals of an infinitesimal duration. This method was demonstrated to be effective in the suppression of spikes in coupled Adler [22] and in two coupled FitzHugh-Nagumo [21] systems. It consists in applying a reset term $V_{c} \delta\left(t-t^{\prime}\right)$ with small fixed amplitude $V_{c}$ at a time instant $t=t^{\prime}$. Here, $\delta\left(t-t^{\prime}\right)$ is the Dirac delta function and $t^{\prime}$ is the time at which the distance $y_{\perp}$ reaches a predefined threshold $\mathcal{T}_{2}$. This reset is applied to the variable $V_{S_{1}}$ after the threshold has been reached. As the goal is to reduce $\left|V_{M_{1}}-V_{S_{1}}\right|$, the reset acts as $V_{S_{1}}\left(t^{\prime}\right) \rightarrow V_{S_{1}}\left(t^{\prime}\right)+V_{c}$ if $V_{M_{2}}>V_{S_{2}}$, or $V_{S_{1}}\left(t^{\prime}\right) \rightarrow V_{S_{1}}\left(t^{\prime}\right)-V_{c}$ if $V_{M_{2}}<V_{S_{2}}$. So we must know simultaneously the predicted variable $y_{\perp}$ and the sign of the difference $V_{M_{2}}-V_{S_{2}}$. In order to avoid additional triggers in a single event 7 units of time (175 $\mu$ s) must pass before the corrective reset can act again.

A typical time trace is shown in Fig. 4(a), where the threshold is fixed to $\mathcal{T}_{2}=0.5$. When the value of the distance $y_{\perp}$ of the auxiliary system crosses the threshold, the control is triggered. As a result, $x_{\perp}$ rarely reaches or surpasses $\mathcal{T}_{2}$. As shown in Figs. 4(b)-4(d), the control efficiently brings $V_{S_{1}}$ 

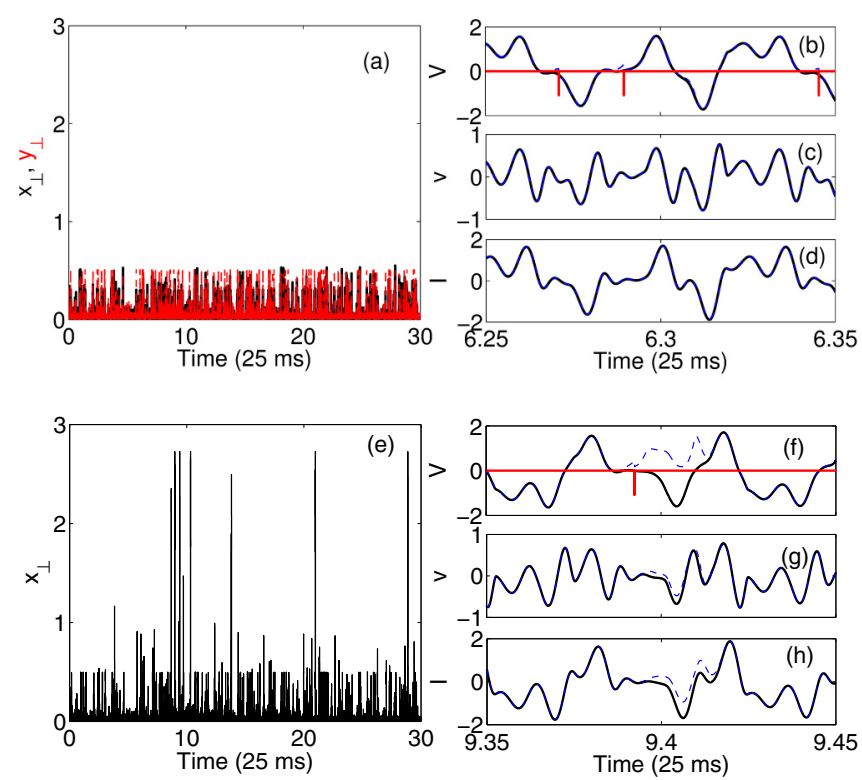

FIG. 4. (Color online) (a) Time trace for $x_{\perp}$ (solid black line) and $y_{\perp}$ (dashed red line). The control is triggered when $y_{\perp}$ exceeds the threshold $\mathcal{T}_{2}=0.5$. (b)-(d) Time trace of each of the variables of the main system $M_{1}$ (solid black line) and $S_{1}$ (dashed blue line). In (b) we also show a magnification of the control [solid red (gray) line]. (e) Time trace for the $x_{\perp}$ setting where the control turns on when $x_{\perp}$ exceeds the threshold $\mathcal{T}_{1}=0.5$. (f)-(h) Time trace of each of the variables of the main system $M_{1}$ (solid black line) and $S_{1}$ (dashed blue line) for a threshold $\mathcal{T}_{1}=0.5$. In (f) we also show a magnification of the control [solid red (gray) line]. $\left|V_{c}\right|=0.27$.

to the evolution of $V_{M_{1}}$ and the initially diverging trajectories converge again after the reset.

For comparison, we can also apply the corrective resets when $x_{\perp}$ reaches a predefined threshold $\mathcal{T}_{1}$. A time trace is shown in Fig. 4(e) for the same set of parameters used in the previous case but now triggering the control when $x_{\perp}$ reaches the value $\mathcal{T}_{1}$. At variance with the case where the reset was triggered by the auxiliary system, i.e., when $y_{\perp}$ reached the value $\mathcal{T}_{2}, x_{\perp}$ can now surpass the threshold value $\mathcal{T}_{1}$ and eventually it keeps growing even after the reset is applied [Figs. 4(f)-4(h)]. By comparing Figs. 4(a) and 4(e) we see that almost complete suppression of extreme events is obtained for a control defined through the predicted variable $y_{\perp}$ crossing the threshold $\mathcal{T}_{2}$. However, for the control defined through $x_{\perp}$ crossing the threshold $\mathcal{T}_{1}$ some extreme events can still be observed in the main system. The corrective reset becomes less effective for a control based on the threshold $\mathcal{T}_{1}$ since $V_{c}$ is too small, resulting in a poor control over the main system. Consequently, anticipated synchronization improves the performance of the control by applying corrective resets of small amplitude a given time in advance.

We have explored a broad range of parameters using even larger mismatches and in all cases the control through the auxiliary system was better. Here, a better control means that we can suppress the extreme events with voltages $V_{c} 30 \%-40 \%$ smaller applied during the same time. To show this, we now calculate the fraction of DKs that survive the control. A dragon king is precisely defined as a desynchronization event that

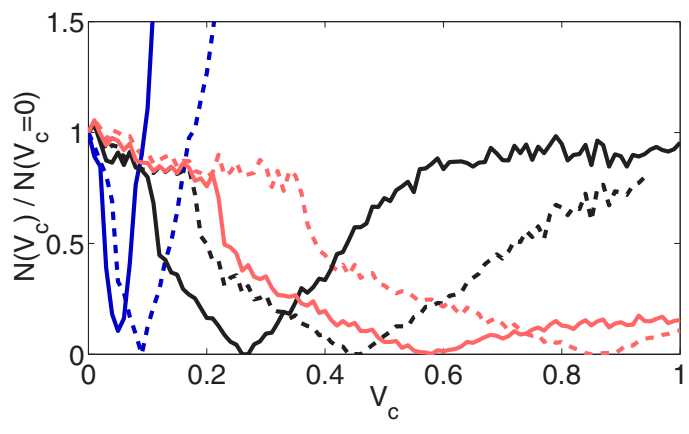

FIG. 5. (Color online) Fraction of detected DKs for a reset amplitude $V_{c}$ divided by the number of DKs when the control is off. The reset is triggered when $x_{\perp}=\mathcal{T}_{1}$ (dashed lines) or when $y_{\perp}=\mathcal{T}_{2}$ (solid lines). The values of the thresholds are from left to right: $\mathcal{T}_{2}=0.1$ [solid blue (dark gray) line], $\mathcal{T}_{1}=0.1$ [dashed blue (dark gray) line], $\mathcal{T}_{2}=0.5$ (solid black line), $\mathcal{T}_{1}=0.5$ (dashed black line), $\mathcal{T}_{2}=1$ [solid red (light gray) line], and $\mathcal{T}_{1}=1$ [dashed red (light gray) line].

increases above $x_{\perp}=2$ and lasts until $x_{\perp}<0.5$. In Fig. 5 we show the fraction of surviving DKs, defined as the number $N$ of DKs detected after the control divided by the number of DKs detected without control, $N\left(V_{c}=0\right)$, for different values of the thresholds $\mathcal{T}_{1}$ and $\mathcal{T}_{2}$. The voltage $V_{c}$ is considered optimal when the fraction of surviving DKs is minimum. The optimal $V_{c}$ is $30 \%-40 \%$ smaller when using the threshold $\mathcal{T}_{2}$ on the auxiliary system (solid lines) than when using the threshold $\mathcal{T}_{1}$ on the main system (dashed lines). Moreover, if we use the threshold $\mathcal{T}_{1}$ and the reset amplitude is fixed, e.g., at $V_{c} \sim 0.25$ (the optimal value for the control using a threshold at $\mathcal{T}_{2}=0.5$ in the auxiliary system), $30 \%-40 \%$ of the DKs remain. It is worth mentioning that above the optimal voltage the fraction of remaining DKs grows with $V_{c}$, the reason being that resets of too large amplitude $V_{c}$ can change the sign of $V_{M_{2}}-V_{S_{2}}$.

If one wants to be more selective and act only on the largest desynchronization events, the corrective resets must be applied at larger threshold values, requiring large voltages $V_{c}$, i.e., more energy, to efficiently suppress the event. The selected threshold also affects the sensitivity to variations of $V_{c}$ due to the $\mathrm{V}$ shape around the optimal $V_{c}$. Large thresholds lead to broader ranges of high suppression [black and red (light gray) lines] while the number of surviving DKs is more sensitive to variations of $V_{c}$ for small thresholds [blue (dark gray) line].

The number of corrective resets is obtained for realizations of a fixed total time ( $t=8 \times 10^{5}$ units of time) and shown in Fig. 6 as a function of $V_{c}$. This value is much larger for small thresholds $\mathcal{T}_{1,2}=0.1$ [blue (dark gray) lines] than for large thresholds [black and red (light gray) lines] as expected from the power-law distribution of desynchronization events. Low thresholds have the inconvenience of triggering the control for a large number of events that do not lead to extreme events. Larger thresholds, $\mathcal{T}_{1,2}=0.5$ and $\mathcal{T}_{1,2}=1$, lead to a small number of resets in the range of optimal control. Note also that for $V_{c}=0$ and $\mathcal{T}_{1,2}=0.1$ [blue (dark gray) lines] the number of resets is different. The distance $y_{\perp}$ crosses the threshold more often than the distance $x_{\perp}$ because of the different parameters used in the main and the auxiliary systems (see Table I). 


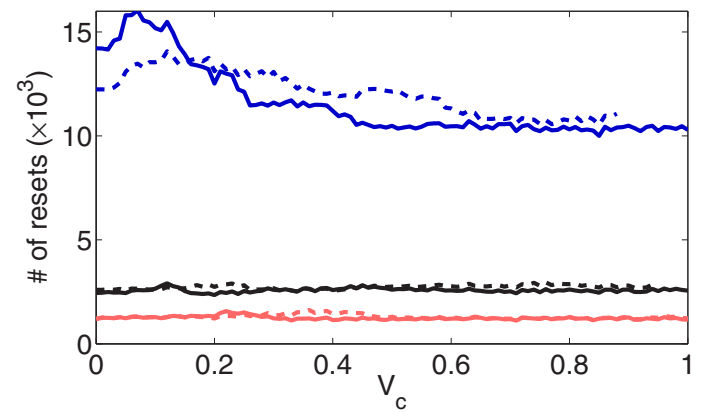

FIG. 6. (Color online) Number of control resets as a function of $V_{c}$. The reset is triggered when $x_{\perp}=\mathcal{T}_{1}$ (dashed lines) or when $y_{\perp}=$ $\mathcal{T}_{2}$ (solid lines) where the values of the thresholds are from top to bottom $\mathcal{T}_{1}=0.1$ [dashed blue (dark gray) line], $\mathcal{T}_{2}=0.1$ [solid blue (dark gray) line], $\mathcal{T}_{1}=0.5$ (dashed black line), $\mathcal{T}_{2}=0.5$ (solid black line), $\mathcal{T}_{1}=1$ [dashed red (light gray) line], and $\mathcal{T}_{2}=1$ [solid red (light gray) line].

\section{B. Stochastic extreme events induced by noise}

Bubbling events can also appear due to noise even though $M_{1}$ and $S_{1}$ are identical. Desynchronization events cannot be predicted just by knowing the current state of the system due to the unpredictability of noise. However, here we show that even in the case of DKs induced by noise, we are able to predict the appearance of such DKs with a certain anticipation time and apply the control method explained in the previous section to prevent the extreme events.

According to the previous description, we set all the parameters identical to the parameters of $M_{1}$ listed in Table I and introduce independent white noises of zero mean and $\delta$ correlated in time to each variable $\zeta_{j}, \xi_{j}$, and $\eta_{j}$, but with the same intensity $D$, i.e., the equations for the main system are modified from Eqs. (3)-(8) such that the master becomes

$$
\begin{gathered}
\dot{V}_{M_{1}}=\frac{V_{M_{1}}}{R_{1, M_{1}}}-g_{M_{1}}\left[V_{M_{1}}-v_{M_{1}}\right]+D \zeta_{M_{1}}, \\
\dot{v}_{M_{1}}=g_{M_{1}}\left[V_{M_{1}}-v_{M_{1}}\right]-I_{M_{1}}+D \xi_{M_{1}}, \\
\dot{I}_{M_{1}}=v_{M_{1}}-R_{4, M_{1}} I_{M_{1}}+D \eta_{M_{1}},
\end{gathered}
$$

and the slave is given by

$$
\begin{gathered}
\dot{V}_{S_{1}}=\frac{V_{S_{1}}}{R_{1, M_{1}}}-g_{M_{1}}\left[V_{S_{1}}-v_{S_{1}}\right]+D \zeta_{S_{1}} \\
\dot{v}_{S_{1}}=g_{M_{1}}\left[V_{S_{1}}-v_{S_{1}}\right]-I_{S_{1}}+\kappa\left(v_{M_{1}}-v_{S_{1}}\right)+D \xi_{S_{1}}, \\
\dot{I}_{S_{1}}=v_{S_{1}}-R_{4, M_{1}} I_{S_{1}}+D \eta_{S_{1}} .
\end{gathered}
$$

Similarly, the auxiliary system is modified from Eqs. (11)(16). The evolution equations for the master of the auxiliary system can be written as

$$
\begin{aligned}
\dot{V}_{M_{2}}= & \frac{V_{M_{2}}}{R_{1, M_{1}}}-g_{M_{1}}\left[V_{M_{2}}-v_{M_{2}}\right] \\
& +C\left[V_{M_{1}}-V_{M_{2}}(t-\tau)\right]+D \zeta_{M_{2}}, \\
\dot{v}_{M_{2}}= & g_{M_{1}}\left[V_{M_{2}}-v_{M_{2}}\right]-I_{M_{2}}+D \xi_{M_{2}},
\end{aligned}
$$

$$
\dot{I}_{M_{2}}=v_{M_{2}}-R_{4, M_{1}} I_{M_{2}}+D \eta_{M_{2}},
$$

and the slave of the auxiliary system becomes

$$
\begin{gathered}
\dot{V}_{S_{2}}=\frac{V_{S_{2}}}{R_{1, M_{1}}}-g_{M_{1}}\left[V_{S_{2}}-v_{S_{2}}\right] \\
+C\left[V_{S_{1}}-V_{S_{2}}(t-\tau)\right]+D \zeta_{S_{2}}, \\
\dot{v}_{S_{2}}=g_{M_{1}}\left[V_{S_{2}}-v_{S_{2}}\right]-I_{S_{2}}+\kappa\left(v_{M_{2}}-v_{S_{2}}\right) \\
+D \xi_{S_{2}}, \\
\dot{I}_{S_{2}}=v_{S_{2}}-R_{4, M_{1}} I_{S_{2}}+D \eta_{S_{2}},
\end{gathered}
$$

where the coupling is introduced in both systems as was done in Sec. II. For $D=0, M_{1}$ and $S_{1}$ are synchronized at all times, i.e., $x_{\perp}=0$. For $D>0$ noise introduces small perturbations that can be amplified in certain regions of the phase space, leading to a bubbling event. The amplitude and frequency of these events depend on the size of the perturbation and the region of the phase space where noise acts.

Now, we apply the same control as in the deterministic case. Interestingly, the ratio of surviving stochastic DKs as a function of $V_{c}$ is comparable to that in the deterministic case as shown in Fig. 7, which suggests that noise plays a role similar to that of the parameter mismatch [24].

The fraction of surviving DKs after the reset is shown in Fig. 8 as a function of the noise strength. If the threshold is defined in $x_{\perp}$ (open and filled squares) the noise strength has almost no effect on the fraction of DKs, which remains around $40 \%$. However, when the threshold is defined in $y_{\perp}$ (open and filled circles) the fraction of surviving DKs can be substantially smaller. For small values of $D$ the fraction is almost zero and monotonically increases when increasing $D$. This is consistent with the results obtained with different sets of parameters in the deterministic case. In Fig. 3 the accuracy of the prediction, as well as the anticipation time, depend on the set of parameters used in the system. When the parameters are very different the region of stable anticipated synchronization decreases and so

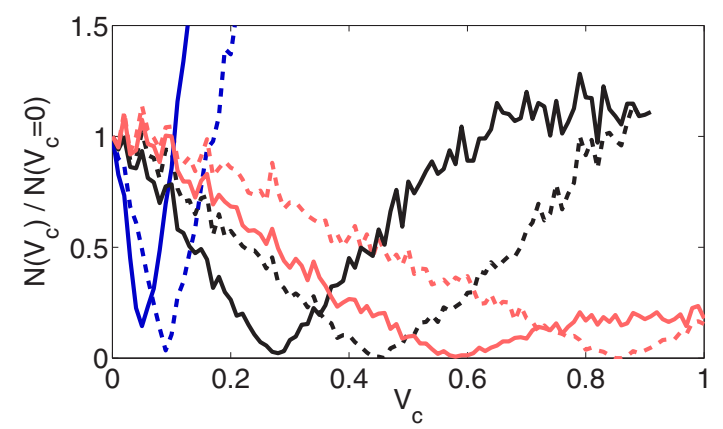

FIG. 7. (Color online) Fraction of detected DKs for a reset amplitude $V_{c}$ divided by the number of DKs when the control is off. The reset is triggered when $x_{\perp}=\mathcal{T}_{1}$ (dashed lines) or when $y_{\perp}=\mathcal{T}_{2}$ (solid lines). The values of the thresholds are from left to right $\mathcal{T}_{2}=0.1$ [solid blue (dark gray) line], $\mathcal{T}_{1}=0.1$ [dashed blue (dark gray) line], $\mathcal{T}_{2}=0.5$ (solid black line), $\mathcal{T}_{1}=0.5$ (dashed black line), $\mathcal{T}_{2}=1$ [solid red (light gray) line], and $\mathcal{T}_{1}=1$ [dashed red (light gray) line]. $D=3 \times 10^{-3}$. 


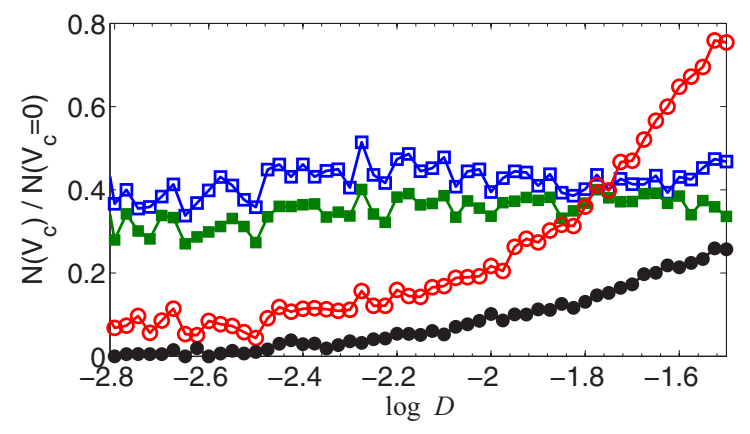

FIG. 8. (Color online) Fraction of detected DKs when the control is on divided by the number of DKs when the control is off as a function of the logarithm of the noise strength $D$. The reset is triggered when $x_{\perp}=\mathcal{T}_{1}$ (squares) or when $y_{\perp}=\mathcal{T}_{2}$ (circles). $\mathcal{T}_{1}=0.2, V_{c}=$ 0.1 (open squares); $\mathcal{T}_{1}=0.5, V_{c}=0.28$ (filled squares); $\mathcal{T}_{2}=0.2$, $V_{c}=0.1$ (open circles); and $\mathcal{T}_{2}=0.5, V_{c}=0.28$ (filled circles).

does the anticipation time. In the stochastic case, we find that the region of anticipated synchronization decreases smoothly on increasing $D$. The quality of the prediction strongly affects the efficiency of the control using anticipated synchronization and suggests that the anticipation method would not be a good option when the extreme events are induced by a large noise.

\section{CONCLUSIONS}

We have shown that anticipated synchronization can be used to predict the occurrence of extreme desynchronization events known as dragon kings in unidirectionally coupled dynamical systems. We have shown that a corrective reset can be combined with anticipated synchronization to efficiently reduce and almost completely suppress these extreme events. In the case of deterministic DKs induced by parameter mismatches, the detection of desynchronization events in the predicted signal requires smaller amplitudes of the resets, when compared with a direct detection in the main system, in order to suppress the DKs. We have also shown that noise can induce DKs, playing a role similar to that of the parameter mismatch. In this case also, anticipated synchronization can be efficiently used to suppress noise-induced DKs if the noise strength is not too large.

Other techniques could also be considered in order to extend the range of applicability of the proposed method. We suggest that anticipated synchronization can be combined with other detection methods in order to achieve larger anticipation times. One recent example relies on the detection of a well-defined precursor before the appearance of an extreme event,

as was shown in semiconductor lasers [12] and in the electronic oscillators used here [16]. Moreover, the auxiliary system could also be replaced by a neural network as an approximate replica of the main system [25] that is particularly useful when the details of the system, e.g., the functional form, are unknown. In case the functional form of the master dynamics is somehow known, certain techniques for parameter adjustment using the time series of the master dynamics can also be useful (see, e.g., [26] and references therein). Recently, a novel modeling framework based on Boolean delayed equations has also been proposed as a heuristic approach to the main dynamical properties of some systems too complex to be modeled with the standard techniques, such as those found in seismicity or in the El Niño and southern oscillations [27]. Boolean delayed equations may be suitably combined with anticipated synchronization in order to extend their prediction horizon, although this requires further exploration to determine their applicability.

\section{ACKNOWLEDGMENTS}

This work was supported by MINECO (Spain), Comunitat Autònoma de les Illes Balears, FEDER, and the European Commission under the INTENSE@COSYP Project No. FIS2012-30634
[1] S. Albeverio, V. Jentsch, and H. Kantz, Extreme Events in Nature and Society, The Frontiers Collection (Springer, Heidelberg, 2006).

[2] H. E. Roman, R. A. Siliprandi, C. Dose, and M. Porto, Phys. Rev. E 80, 036114 (2009).

[3] Richard W. Katz, Climatic Change 100, 71 (2010).

[4] D. R. Solli, C. Ropers, P. Koonath, and B. Jalali, Nature (London) 450, 1054 (2007).

[5] A. K. Dal Bosco, D. Wolfersberger and M. Sciamanna, Opt. Lett. 38, 703 (2013).

[6] V. I. Yukalov, E. P. Yukalova, and D. Sornette, Eur. Phys. J.: Spec. Top. 205, 313 (2012).

[7] C. Nicolis, V. Balakrishnan, and G. Nicolis, Phys. Rev. Lett. 97, 210602 (2006).

[8] D. Sornette, Int. J. Terraspace Sci. Eng. 2, 1 (2009).

[9] D. Sornette and G. Ouillon, Eur. Phys. J.: Spec. Top. 205, 1 (2012).

[10] C. Kharif, E. Pelinovsky, and A. Slunyaev, Rogue Waves in the Ocean (Springer, Heidelberg, 2009).
[11] C. Bonatto, M. Feyereisen, S. Barland, M. Giudici, C. Masoller, José R. Rios Leite, and J. R. Tredicce, Phys. Rev. Lett. 107, 053901 (2011).

[12] J. Zamora-Munt, B. Garbin, S. Barland, M. Giudici, Jose R. Rios Leite, C. Masoller, and J. R. Tredicce, Phys. Rev. A 87, 035802 (2013).

[13] V. Flunkert, O. DHuys, J. Danckaert, I. Fischer, and E. Schöll, Phys. Rev. E 79, 065201 (2009).

[14] J. Tiana-Alsina, K. Hicke, X. Porte, M. C. Soriano, M. C. Torrent, J. García-Ojalvo, and I. Fischer, Phys. Rev. E 85, 026209 (2012).

[15] D. J. Gauthier and J. C. Bienfang, Phys. Rev. Lett. 77, 1751 (1996).

[16] H. L. D. de S. Cavalcante, M. Oriá, D. Sornette, E. Ott, and D. J. Gauthier, Phys. Rev. Lett. 111, 198701 (2013).

[17] H. U. Voss, Phys. Rev. E 61, 5115 (2000).

[18] C. Masoller, Phys. Rev. Lett. 86, 2782 (2001).

[19] S. Sivaprakasam, E. M. Shahverdiev, P. S. Spencer, and K. A. Shore, Phys. Rev. Lett. 87, 154101 (2001). 
[20] M. Ciszak, O. Calvo, C. Masoller, C. R. Mirasso, and R. Toral, Phys. Rev. Lett. 90, 204102 (2003).

[21] M. Ciszak, C. R. Mirasso, R. Toral, and O. Calvo, Phys. Rev. E 79, 046203 (2009).

[22] C. Mayol, C. R. Mirasso, and R. Toral, Phys. Rev. E 85, 056216 (2012).

[23] The delay time for which anticipated synchronization occurs, e.g., see Fig. 2, is relatively small compared with the time scale of the extreme events and is smaller than in other systems. We speculate that this is due to the strong nonlinearities of the system and expect larger anticipation times in systems with weaker nonlinearities.

[24] C. J. Tessone, A. Scirè, R. Toral, and P. Colet, Phys. Rev. E 75, 016203 (2007).

[25] M. Ciszak, J. M. Gutiérrez, A. S. Cofiño, C. Mirasso, R. Toral, L. Pesquera, and S. Ortín, Phys. Rev. E 72, 046218 (2005).

[26] H. Abarbanel, D. Creveling, R. Farsian, and M. Kostuk, SIAM J. Appl. Dyn. Syst. 8, 1341 (2009).

[27] M. Ghil et al., Nonlin. Process. Geophys. 18, 295 (2011). 